Bull. Korean Math. Soc. 51 (2014), No. 1, pp. 99-113

http://dx.doi.org/10.4134/BKMS.2014.51.1.099

\title{
MULTIPLE SOLUTIONS FOR A $p$-LAPLACIAN SYSTEM WITH NONLINEAR BOUNDARY CONDITIONS
}

\author{
Jun Zhou and Chan-Gyun Kim
}

\begin{abstract}
A nonlinear elliptic problem involving $p$-Laplacian and nonlinear boundary condition is considered in this paper. By using the method of Nehari manifold, it is proved that the system possesses two nontrivial nonnegative solutions if the parameter is small enough.
\end{abstract}

\section{Introduction}

This paper is devoted to the study of the following quasilinear elliptic problem with nonlinear boundary conditions:

$$
\begin{cases}-\Delta_{p} u+m(x)|u|^{p-2} u=\lambda F_{u}(u, v), & x \in \Omega, \\ -\Delta_{p} v+n(x)|v|^{p-2} v=\lambda F_{v}(u, v), & x \in \Omega, \\ |\nabla u|^{p-2} \frac{\partial u}{\partial \nu}=G_{u}(u, v),|\nabla v|^{p-2} \frac{\partial v}{\partial \nu}=G_{v}(u, v), & x \in \partial \Omega,\end{cases}
$$

where $p>1, \Omega \subset \mathbb{R}^{N}$ is a bounded domain with smooth boundary $\partial \Omega$ and

- $\Delta_{p}$ denotes the $p$-Laplacian operator, defined by $\Delta_{p} z=\operatorname{div}\left(|\nabla z|^{p-2} \nabla z\right)$;

- $\lambda \in(0,+\infty), m(x), n(x) \in C(\bar{\Omega})$, and there exist positive constants $m_{0}$ and $n_{0}$ such that $m(x) \geq m_{0}$ and $n(x) \geq n_{0}$ for all $x \in \bar{\Omega}$;

- $\nu$ is the unit outer normal to $\partial \Omega$;

- $F, G: \mathbb{R} \times \mathbb{R} \rightarrow[0, \infty)$ satisfy:

(H1) $F, G \in C^{1}(\mathbb{R} \times \mathbb{R}) ; F(u, v)=F(|u|,|v|)$ and $G(u, v)=G(|u|,|v|)$; $F(u, v) \not \equiv 0$ and $G(u, v) \not \equiv 0$;

(H2) There exist constants $\alpha \in\left(p, p^{*}\right)$ and $\beta \in(1, p)$ such that

$$
F(t u, t v)=t^{\alpha} F(u, v) \text { and } G(t u, t v)=t^{\beta} G(u, v)
$$

for all $t \geq 0$ and $(u, v) \in \mathbb{R} \times \mathbb{R}$.

Received November 14, 2012

2010 Mathematics Subject Classification. 35J50, 35J57, 35J66.

Key words and phrases. p-Laplacian systems, nonlinear boundary condition, multiple solutions, Nehari manifold. 
Here $p^{*}$ denotes the Sobolev conjugate exponent of $p$, i.e.,

$$
p^{*}= \begin{cases}\frac{N p}{N-p}, & p<N, \\ \infty, & p \geq N .\end{cases}
$$

Problem involving the $p$-Laplacian operator appears in pure mathematics such as the theory of quasiregular and quasiconformal mapping [26, 39] as well as in applied mathematics. Indeed, it intervenes in numerous fields in experimental sciences: nonlinear reaction-diffusion problems, dynamics of populations, non-Newtonian fluids, flows through porous media, nonlinear elasticity, petroleum extraction, torsional creep problems, etc (see, e.g., [21, 22, 42]). In literature, there exist numerous papers dedicated to the study of such equations and systems. In fact, the study of scalar equations had really started in the middle of 80 s by M. Ôtani [34] in one dimension and then in dimension $N$ by F. de Thélin [19]. Later, the results are generalized to other kinds of equations or systems involving $p$-Laplacian in $\mathbb{R}^{N}$ or bounded open set $\Omega \subset \mathbb{R}^{N}$ (see, e.g., $[1,3,4,5,6,7,8,13,18,20,23,24,27,29,30,33,35]$ and the references therein).

In recent years, the existence of solutions for the semilinear/quasilinear elliptic equations with nonlinear boundary conditions have been widely studied (see, e.g., $[9,12,16,17,28,36,37,41]$ and the references therein). In particular, in [37], the authors studied the multiple solutions of the following systems:

$$
\begin{cases}-\Delta_{p} u+m(x)|u|^{p-2} u=\lambda_{1} a(x)|u|^{\gamma-2} u, & x \in \Omega \\ -\Delta_{p} v+m(x)|v|^{p-2} v=\lambda_{2} b(x)|v|^{\gamma-2} v, & x \in \Omega \\ |\nabla u|^{p-2} \frac{\partial u}{\partial \nu}=\frac{\alpha}{\alpha+\beta}|u|^{\alpha-2} u|v|^{\beta},|\nabla v|^{p-2} \frac{\partial v}{\partial \nu}=\frac{\beta}{\alpha+\beta}|u|^{\alpha}|v|^{\beta-2} v, & x \in \partial \Omega\end{cases}
$$

where $\Omega \subset \mathbb{R}^{N}, p>2$, is a bounded domain with smooth boundary $\partial \Omega$, $\lambda_{1}, \lambda_{2}>0$, and $2<\alpha+\beta<p<\gamma<p^{*}$. Motivated by the results of the above works, we are interested in the existence of multiple nontrivial nonnegative solutions for problem (1.1). We remark that problem (1.2) is a special case of (1.1) with

$$
F(u, v)=\frac{\lambda_{1}}{\lambda \gamma} a(x)|u|^{\gamma}+\frac{\lambda_{2}}{\lambda \gamma} b(x)|v|^{\gamma}, G(u, v)=\frac{1}{\alpha+\beta}|u|^{\alpha}|v|^{\beta}, \lambda=\lambda_{1}+\lambda_{2} .
$$

The main approach of this paper is the method of Nehari manifold, which was first introduced by Nehari in [31,32], and the method turned out to be very useful in critical point theory (see, e.g., $[1,2,10,11,14,15,25,37,38,40,41]$ ) and eventually came to bear his name.

The rest of this work is organized as follows. In Section 2, we introduce some preliminaries including definitions and some lemmas for later use. In Section 3 , the proof of the main result is given. 


\section{Preliminaries}

Let $W=W^{1, p}(\Omega) \times W^{1, p}(\Omega)$ be a Banach space with norm

$$
\|(u, v)\|=\left(\int_{\Omega}\left(|\nabla u|^{p}+m(x)|u|^{p}\right) d x+\int_{\Omega}\left(|\nabla v|^{p}+n(x)|v|^{p}\right) d x\right)^{1 / p} .
$$

Definition 2.1. We say that $(u, v) \in W$ is a solution to (1.1) if for any $(\phi, \varphi) \in W$

$$
\begin{aligned}
& \int_{\Omega}\left(|\nabla u|^{p-2} \nabla u \cdot \nabla \phi+m(x)|u|^{p-2} u \phi\right) d x=\lambda \int_{\Omega} F_{u}(u, v) \phi d x+\int_{\partial \Omega} G_{u}(u, v) \phi d s \\
& \int_{\Omega}\left(|\nabla v|^{p-2} \nabla v \cdot \nabla \varphi+n(x)|v|^{p-2} v \varphi\right) d x=\lambda \int_{\Omega} F_{v}(u, v) \varphi d x+\int_{\partial \Omega} G_{v}(u, v) \varphi d s .
\end{aligned}
$$

Let $\mathcal{J}_{\lambda}: W \rightarrow \mathbb{R}$ be the corresponding energy functional to problem (1.1) defined as

$$
\mathcal{J}_{\lambda}(u, v)=\frac{1}{p}\|(u, v)\|^{p}-\lambda \int_{\Omega} F(u, v) d x-\int_{\partial \Omega} G(u, v) d s, \quad(u, v) \in W .
$$

Furthermore the nonnegative solutions of problem (1.1) correspond to the critical points of $\mathcal{J}$. Define $I_{\lambda}: W \rightarrow \mathbb{R}$ as

$$
I_{\lambda}(u, v)=\|(u, v)\|^{p}-\lambda \alpha \int_{\Omega} F(u, v) d x-\beta \int_{\partial \Omega} G(u, v) d s, \quad(u, v) \in W .
$$

It follows from condition (H2) that $F_{u}(u, v) u+F_{v}(u, v) v=\alpha F(u, v)$ and $G_{u}(u, v) u+G_{v}(u, v) v=\beta G(u, v)$ for all $u, v \in \mathbb{R}$. Consequently,

$$
I_{\lambda}(u, v)=\left\langle\mathcal{J}_{\lambda}^{\prime}(u, v),(u, v)\right\rangle \text { for all }(u, v) \in W .
$$

Let us denote the Nehari manifold by $\mathcal{N}_{\lambda}$, i.e.,

$$
\mathcal{N}_{\lambda}=\left\{(u, v) \in W \backslash\{(0,0)\}: I_{\lambda}(u, v)=0\right\} .
$$

It is easy to see that $(u, v) \in \mathcal{N}_{\lambda}$ if and only if

$$
\|(u, v)\|^{p}=\lambda \alpha \int_{\Omega} F(u, v) d x+\beta \int_{\partial \Omega} G(u, v) d s .
$$

Accordingly, for $(u, v) \in \mathcal{N}_{\lambda}$,

$$
\begin{aligned}
\left\langle I_{\lambda}^{\prime}(u, v),(u, v)\right\rangle & =p\|(u, v)\|^{p}-\lambda \alpha^{2} \int_{\Omega} F(u, v) d x-\beta^{2} \int_{\partial \Omega} G(u, v) d s \\
& =-(\alpha-p)\|(u, v)\|^{p}+\beta(\alpha-\beta) \int_{\partial \Omega} G(u, v) d s \\
& =(p-\beta)\|(u, v)\|^{p}-\lambda \alpha(\alpha-\beta) \int_{\Omega} F(u, v) d x .
\end{aligned}
$$

By (H2), $F$ and $G$ satisfy that, for all $u, v \in \mathbb{R}$,

$$
F(u, v) \leq M\left(|u|^{p}+|v|^{p}\right)^{\frac{\alpha}{p}}, G(u, v) \leq M\left(|u|^{p}+|v|^{p}\right)^{\frac{\beta}{p}},
$$


where

$$
M:=\max \left\{\max _{|u|^{p}+|v|^{p}=1} F(u, v), \max _{|u|^{p}+|v|^{p}=1} G(u, v)\right\}>0 .
$$

Since $\alpha \in\left(1, p^{*}\right)$ and $\beta \in(1, p)$, it follows from Sobolev and Sobolev trace inequalities that there exist positive constants $C_{1}$ and $C_{2}$ such that

$$
\int_{\Omega}\left(|u|^{p}+|v|^{p}\right)^{\alpha / p} d x \leq C_{1}\|(u, v)\|^{\alpha}, \int_{\partial \Omega}\left(|u|^{p}+|v|^{p}\right)^{\beta / p} d s \leq C_{2}\|(u, v)\|^{\beta}
$$

for all $(u, v) \in W$. By $(2.3)$ and (2.4),

$$
\int_{\Omega} F(u, v) d x \leq M C_{1}\|(u, v)\|^{\alpha}
$$

and

$$
\int_{\partial \Omega} G(u, v) d s \leq M C_{2}\|(u, v)\|^{\beta}
$$

for all $(u, v) \in W$.

Define

$$
\lambda^{*}:=\left(\frac{p-\beta}{\alpha(\alpha-\beta) M C_{1}}\right)\left(\frac{\alpha-p}{\beta(\alpha-\beta) M C_{2}}\right)^{\frac{\alpha-p}{p-\beta}}
$$

and

$$
\lambda_{*}:=\left(\frac{p-\beta}{\alpha(\alpha-\beta) M C_{1}}\right)\left(\frac{\alpha-p}{p(\alpha-\beta) M C_{2}}\right)^{\frac{\alpha-p}{p-\beta}},
$$

where $M$ is the constant in (2.3) and $C_{1}, C_{2}$ are the constants in (2.4). Note that $0<\lambda_{*}<\lambda^{*}$.

Now we split $\mathcal{N}_{\lambda}$ into three parts:

$$
\begin{aligned}
& \mathcal{N}_{\lambda}^{+}=\left\{(u, v) \in \mathcal{N}_{\lambda}:\left\langle I_{\lambda}^{\prime}(u, v),(u, v)\right\rangle>0\right\}, \\
& \mathcal{N}_{\lambda}^{0}=\left\{(u, v) \in \mathcal{N}_{\lambda}:\left\langle I_{\lambda}^{\prime}(u, v),(u, v)\right\rangle=0\right\}, \\
& \mathcal{N}_{\lambda}^{-}=\left\{(u, v) \in \mathcal{N}_{\lambda}:\left\langle I_{\lambda}^{\prime}(u, v),(u, v)\right\rangle<0\right\},
\end{aligned}
$$

and present some properties of $\mathcal{N}_{\lambda}$.

Lemma 2.2. Suppose that $F$ and $G$ satisfy $(\mathrm{H} 1)$ and $(\mathrm{H} 2)$. Then $\mathcal{N}_{\lambda}^{0}=\emptyset$ for all $\lambda \in\left(0, \lambda^{*}\right)$.

Proof. Let $\lambda$ be a fixed number satisfying $\mathcal{N}_{\lambda}^{0} \neq \emptyset$. Then for $(u, v) \in \mathcal{N}_{\lambda}^{0}$,

$$
\begin{aligned}
0=\left\langle I_{\lambda}^{\prime}(u, v),(u, v)\right\rangle & =(p-\beta)\|(u, v)\|^{p}+\lambda \alpha(\beta-\alpha) \int_{\Omega} F(u, v) d x \\
& =(p-\alpha)\|(u, v)\|^{p}+\beta(\alpha-\beta) \int_{\partial \Omega} G(u, v) d s .
\end{aligned}
$$

By (2.5), (2.6) and (2.7),

$$
\left(\frac{p-\beta}{\lambda \alpha(\alpha-\beta) M C_{1}}\right)^{\frac{1}{\alpha-p}} \leq\|(u, v)\| \leq\left(\frac{\beta(\alpha-\beta) M C_{2}}{\alpha-p}\right)^{\frac{1}{p-\beta}}
$$


and consequently

$$
\lambda \geq\left(\frac{p-\beta}{\alpha(\alpha-\beta) M C_{1}}\right)\left(\frac{\alpha-p}{\beta(\alpha-\beta) M C_{2}}\right)^{\frac{\alpha-p}{p-\beta}}=\lambda^{*} .
$$

Lemma 2.3. Suppose that $F$ and $G$ satisfy (H1) and (H2), and $\lambda \in\left(0, \lambda^{*}\right)$. Assume that $\left(u_{0}, v_{0}\right)$ is a local minimizer for $\mathcal{J}_{\lambda}$ on $\mathcal{N}_{\lambda}$. Then $\left(u_{0}, v_{0}\right)$ is a critical point of $\mathcal{J}_{\lambda}$, i.e., $\mathcal{J}_{\lambda}^{\prime}\left(u_{0}, v_{0}\right)=0$.

Proof. Let $\left(u_{0}, v_{0}\right)$ be a local minimizer for $\mathcal{J}_{\lambda}$ on $\mathcal{N}_{\lambda}$ and $\lambda \in\left(0, \lambda^{*}\right)$. Then $\left(u_{0}, v_{0}\right)$ is a solution of the following optimization problem:

$$
\text { minimize } \mathcal{J}_{\lambda}(u, v)=0 \text { subject to } I_{\lambda}(u, v)=0 .
$$

Hence, by the theory of Lagrange multipliers, there exists a $\Lambda \in \mathbb{R}$ such that

$$
\mathcal{J}_{\lambda}^{\prime}\left(u_{0}, v_{0}\right)=\Lambda I_{\lambda}^{\prime}\left(u_{0}, v_{0}\right) .
$$

Thus

$$
\left\langle\mathcal{J}_{\lambda}^{\prime}\left(u_{0}, v_{0}\right),\left(u_{0}, v_{0}\right)\right\rangle=\Lambda\left\langle I_{\lambda}^{\prime}\left(u_{0}, v_{0}\right),\left(u_{0}, v_{0}\right)\right\rangle .
$$

Since $\left(u_{0}, v_{0}\right) \in \mathcal{N}_{\lambda},\left\langle\mathcal{J}_{\lambda}^{\prime}\left(u_{0}, v_{0}\right),\left(u_{0}, v_{0}\right)\right\rangle=0$. On the other hand, by Lemma 2.2 ,

$$
\left\langle I_{\lambda}^{\prime}\left(u_{0}, v_{0}\right),\left(u_{0}, v_{0}\right)\right\rangle \neq 0 .
$$

Hence $\Lambda=0$, and this completes the proof.

Lemma 2.4. $\mathcal{J}_{\lambda}$ is coercive and bounded below on $\mathcal{N}_{\lambda}$ for all $\lambda>0$.

Proof. For $u \in \mathcal{N}_{\lambda}$, it follows from (2.1) and (2.6) that

$$
\begin{aligned}
\mathcal{J}_{\lambda}(u, v) & =\frac{\alpha-p}{p \alpha}\|(u, v)\|^{p}-\frac{\alpha-\beta}{\alpha} \int_{\partial \Omega} G(u, v) d s \\
& \geq \frac{\alpha-p}{p \alpha}\|(u, v)\|^{p}-\frac{\alpha-\beta}{\alpha} M C_{2}\|(u, v)\|^{\beta},
\end{aligned}
$$

which completes the proof since $\beta<p<\alpha$.

By Lemma 2.2, $\mathcal{N}_{\lambda}=\mathcal{N}_{\lambda}^{+} \cup \mathcal{N}_{\lambda}^{-}$for $0<\lambda<\lambda^{*}$, and define

$$
\begin{aligned}
& \gamma_{\lambda}^{+}=\inf \left\{\mathcal{J}_{\lambda}(u, v):(u, v) \in \mathcal{N}_{\lambda}^{+}\right\}, \\
& \gamma_{\lambda}^{-}=\inf \left\{\mathcal{J}_{\lambda}(u, v):(u, v) \in \mathcal{N}_{\lambda}^{-}\right\} .
\end{aligned}
$$

Lemma 2.5. Suppose that $F$ and $G$ satisfy (H1) and (H2). Then we have

(i) If $\lambda \in(0,+\infty)$, then $\gamma_{\lambda}^{+}<0$;

(ii) If $\lambda \in\left(0, \lambda_{*}\right), \gamma_{\lambda}^{-} \geq d_{0}$ for some constant $d_{0}=d_{0}(\lambda)>0$.

Proof. (i) Let $(u, v) \in \mathcal{N}_{\lambda}^{+}$and $\lambda \in(0, \infty)$. By $(2.2)$,

$$
\frac{p-\beta}{\alpha(\alpha-\beta)}\|(u, v)\|^{p}>\lambda \int_{\Omega} F(u, v) d x
$$


and, by (2.1),

$$
\begin{aligned}
\mathcal{J}_{\lambda}(u, v) & =\left(\frac{1}{p}-\frac{1}{\beta}\right)\|(u, v)\|^{p}+\left(\frac{\alpha}{\beta}-1\right) \lambda \int_{\Omega} F(u, v) d x \\
& <\left(\frac{1}{p}-\frac{1}{\beta}+\frac{p-\beta}{\alpha \beta}\right)\|(u, v)\|^{p} \\
& =\frac{(p-\alpha)(p-\beta)}{p \alpha \beta}\|(u, v)\|^{p} .
\end{aligned}
$$

Since $\beta<p<\alpha, \mathcal{J}_{\lambda}(u, v)<0$ for all $(u, v) \in \mathcal{N}_{\lambda}^{+}$, and thus $\gamma_{\lambda}^{+}<0$ for all $\lambda \in(0, \infty)$.

(ii) Let $(u, v) \in \mathcal{N}_{\lambda}^{-}$and $\lambda \in\left(0, \lambda_{*}\right)$. By $(2.2)$ and (2.5),

$$
(p-\beta)\|(u, v)\|^{p}<\lambda \alpha(\alpha-\beta) \int_{\Omega} F(u, v) d x \leq \lambda \alpha(\alpha-\beta) M C_{1}\|(u, v)\|^{\alpha},
$$

and

$$
\|(u, v)\|>\left(\frac{p-\beta}{\lambda \alpha(\alpha-\beta) M C_{1}}\right)^{\frac{1}{\alpha-p}} .
$$

By (2.8) and (2.9),

$$
\begin{aligned}
& \mathcal{J}_{\lambda}(u, v) \\
\geq & \|(u, v)\|^{\beta}\left[\frac{\alpha-p}{p \alpha}\|(u, v)\|^{p-\beta}-\frac{\alpha-\beta}{\alpha} M C_{2}\right] \\
> & \left(\frac{p-\beta}{\lambda \alpha(\alpha-\beta) M C_{1}}\right)^{\frac{\beta}{\alpha-p}}\left[\left(\frac{\alpha-p}{p \alpha}\right)\left(\frac{p-\beta}{\lambda \alpha(\alpha-\beta) M C_{1}}\right)^{\frac{p-\beta}{\alpha-p}}-\left(\frac{\alpha-\beta}{\alpha}\right) M C_{2}\right] .
\end{aligned}
$$

Thus, for each $\lambda \in\left(0, \lambda_{*}\right)$, there exists a positive constant $d_{0}=d_{0}(\lambda)$ such that

$$
\gamma_{\lambda}^{-} \geq d_{0}
$$

Lemma 2.6. Suppose that $F$ and $G$ satisfy (H1) and (H2). Let $\lambda \in\left(0, \lambda_{*}\right)$ and $(u, v) \in W$. Then we have

(i) If $\int_{\Omega} F(u, v) d x>0$, there exists a unique $t_{2}=t_{2}(u, v)$ with

$$
t_{2}>t_{1}^{*}=t_{1}^{*}(u, v):=\left[\frac{(p-\beta)\|(u, v)\|^{p}}{\lambda \alpha(\alpha-\beta) \int_{\Omega} F(u, v) d x}\right]^{\frac{1}{\alpha-p}}>0
$$

such that $\left(t_{2} u, t_{2} v\right) \in \mathcal{N}_{\lambda}^{-}$and $\mathcal{J}_{\lambda}\left(t_{2} u, t_{2} v\right)=\sup _{t \geq 0} \mathcal{J}_{\lambda}(t u, t v)$.

(ii) If $\int_{\partial \Omega} G(u, v) d s>0$, there exists a unique $t_{3}=t_{3}(u, v)$ with

$$
0<t_{3}<t_{2}^{*}=t_{2}^{*}(u, v):=\left[\frac{\beta(\alpha-\beta) \int_{\partial \Omega} G(u, v) d s}{(\alpha-p)\|(u, v)\|^{p}}\right]^{\frac{1}{p-\beta}}
$$


such that $\left(t_{3} u, t_{3} v\right) \in \mathcal{N}_{\lambda}^{+}$and $\mathcal{J}_{\lambda}\left(t_{3} u, t_{3} v\right)=\inf _{0 \leq t \leq t_{2}^{*}} \mathcal{J}_{\lambda}(t u, t v)$.

Proof. (i) Fix $(u, v) \in W$ with $\int_{\Omega} F(u, v) d x>0$. Then $(u, v) \neq(0,0)$. Let

$$
a_{(u, v)}(t)=t^{p-\beta}\|(u, v)\|^{p}-\lambda \alpha t^{\alpha-\beta} \int_{\Omega} F(u, v) d x \text { for } t \geq 0 .
$$

Then $a_{(u, v)}(0)=0$ and $a_{(u, v)}(t) \rightarrow-\infty$ as $t \rightarrow+\infty$. Since

$$
a_{(u, v)}^{\prime}(t)=(p-\beta) t^{p-\beta-1}\|(u, v)\|^{p}-\lambda \alpha(\alpha-\beta) t^{\alpha-\beta-1} \int_{\Omega} F(u, v) d x,
$$

we see that $a_{(u, v)}^{\prime}(t)=0$ for $t=t_{1}^{*}, a_{(u, v)}^{\prime}(t)>0$ for $t \in\left(0, t_{1}^{*}\right)$ and $a_{(u, v)}^{\prime}(t)<0$ for $t \in\left(t_{1}^{*},+\infty\right)$. Moreover, by (2.5),

$$
\begin{aligned}
a_{(u, v)}\left(t_{1}^{*}\right) & =\left(t_{1}^{*}\right)^{p-\beta}\|(u, v)\|^{p}\left(\frac{\alpha-p}{\alpha-\beta}\right) \\
& =\left(\frac{(p-\beta)\|(u, v)\|^{\alpha}}{\lambda \alpha(\alpha-\beta) \int_{\Omega} F(u, v) d x}\right)^{\frac{p-\beta}{\alpha-p}}\|(u, v)\|^{\beta}\left(\frac{\alpha-p}{\alpha-\beta}\right) \\
& >\left(\frac{p-\beta}{\lambda_{*} \alpha(\alpha-\beta) M C_{1}}\right)^{\frac{p-\beta}{\alpha-p}}\left(\frac{\alpha-p}{\alpha-\beta}\right)\|(u, v)\|^{\beta} .
\end{aligned}
$$

On the other hand, by (2.6),

$$
\begin{aligned}
0 & \leq \beta \int_{\partial \Omega} G(u, v) d s \\
& \leq \beta M C_{2}\|(u, v)\|^{\beta} \\
& \leq\left(\frac{\alpha-p}{\alpha-\beta}\right)\left(\frac{p-\beta}{\lambda_{*} \alpha(\alpha-\beta) M C_{1}}\right)^{\frac{p-\beta}{\alpha-p}}\|(u, v)\|^{\beta}
\end{aligned}
$$

and, by (2.10),

$$
\beta \int_{\partial \Omega} G(u, v) d s<a_{(u, v)}\left(t_{1}^{*}\right) .
$$

Hence, there are unique $t_{1}=t_{1}(u, v)$ and $t_{2}=t_{2}(u, v)$ such that $0 \leq t_{1}<t_{1}^{*}<t_{2}, a_{(u, v)}\left(t_{1}\right)=a_{(u, v)}\left(t_{2}\right)=\beta \int_{\partial \Omega} G(u, v) d s \quad$ and $a_{(u, v)}^{\prime}\left(t_{2}\right)<0$.

Clearly $\left(t_{2} u, t_{2} v\right) \neq(0,0)$, and $\left(t_{2} u, t_{2} v\right) \in \mathcal{N}_{\lambda}$ since

$$
\begin{aligned}
I_{\lambda}\left(t_{2} u, t_{2} v\right) & =t_{2}^{p}\|(u, v)\|^{p}-\lambda \alpha t_{2}^{\alpha} \int_{\Omega} F(u, v) d x-\beta t_{2}^{\beta} \int_{\partial \Omega} G(u, v) d s \\
& =t_{2}^{\beta}\left(a_{(u, v)}\left(t_{2}\right)-\beta \int_{\partial \Omega} G(u, v) d s\right)=0 .
\end{aligned}
$$

It follows from (2.2) that

$$
\begin{aligned}
\left\langle I_{\lambda}^{\prime}\left(t_{2} u, t_{2} v\right),\left(t_{2} u, t_{2} v\right)\right\rangle & =(p-\beta) t_{2}^{p}\|(u, v)\|^{p}-\lambda \alpha(\alpha-\beta) t_{2}^{\alpha} \int_{\Omega} F(u, v) d x \\
& =t_{2}^{\beta+1} a_{(u, v)}^{\prime}\left(t_{2}\right)<0 .
\end{aligned}
$$


Thus $\left(t_{2} u, t_{2} v\right) \in \mathcal{N}_{\lambda}^{-}$. Moreover

$$
\begin{aligned}
\frac{d}{d t} \mathcal{J}_{\lambda}(t u, t v) & =t^{p-1}\|(u, v)\|^{p}-\lambda \alpha t^{\alpha-1} \int_{\Omega} F(u, v) d x-\beta t^{\beta-1} \int_{\partial \Omega} G(u, v) d s \\
& =t^{\beta-1}\left(a_{(u, v)}(t)-\beta \int_{\partial \Omega} G(u, v) d s\right)
\end{aligned}
$$

which implies that $\frac{d}{d t} \mathcal{J}_{\lambda}(t u, t v)=0$ for $t=t_{1}$ and $t=t_{2} ; \frac{d}{d t} \mathcal{J}_{\lambda}(t u, t v)<0$ for $t \in\left(0, t_{1}\right) \cup\left(t_{2},+\infty\right) ; \frac{d}{d t} \mathcal{J}_{\lambda}(t u, t v)>0$ for $t \in\left(t_{1}, t_{2}\right)$. From Lemma 2.5(ii) and $\mathcal{J}_{\lambda}(0,0)=0$, it follows that

$$
\mathcal{J}_{\lambda}\left(t_{2} u, t_{2} v\right)=\sup _{t \geq 0} \mathcal{J}_{\lambda}(t u, t v)
$$

(ii) Fix $(u, v) \in W$ with $\int_{\partial \Omega} G(u, v) d s>0$. Then $(u, v) \neq(0,0)$. Let

$$
b_{(u, v)}(t)=t^{p-\alpha}\|(u, v)\|^{p}-\beta t^{\beta-\alpha} \int_{\partial \Omega} G(u, v) d s \quad \text { for } t>0 .
$$

Then $b_{(u, v)}(t) \rightarrow-\infty$ as $t \rightarrow 0^{+}$and $b_{(u, v)}(t) \rightarrow 0$ as $t \rightarrow+\infty$. Since

$$
b_{(u, v)}^{\prime}(t)=(p-\alpha) t^{p-\alpha-1}\|(u, v)\|^{p}-\beta(\beta-\alpha) t^{\beta-\alpha-1} \int_{\partial \Omega} G(u, v) d s,
$$

we see that $b_{(u, v)}^{\prime}(t)=0$ for $t=t_{2}^{*}, b_{(u, v)}^{\prime}(t)>0$ for $t \in\left(0, t_{2}^{*}\right)$ and $b_{(u, v)}^{\prime}(t)<0$ for $t \in\left(t_{2}^{*},+\infty\right)$. Moreover, by (2.6),

$$
\begin{aligned}
b_{(u, v)}\left(t_{2}^{*}\right) & =\left(t_{2}^{*}\right)^{p-\alpha}\|(u, v)\|^{p}\left(\frac{p-\beta}{\alpha-\beta}\right) \\
& =\left(\frac{(\alpha-p)\|(u, v)\|^{\beta}}{\beta(\alpha-\beta) \int_{\partial \Omega} G(u, v) d s}\right)^{\frac{\alpha-p}{p-\beta}}\|(u, v)\|^{\alpha}\left(\frac{p-\beta}{\alpha-\beta}\right) \\
& \geq\left(\frac{\alpha-p}{\beta(\alpha-\beta) M C_{2}}\right)^{\frac{\alpha-p}{p-\beta}}\|(u, v)\|^{\alpha}\left(\frac{p-\beta}{\alpha-\beta}\right) .
\end{aligned}
$$

On the other hand, by (2.5),

$$
\begin{aligned}
0 & \leq \lambda \alpha \int_{\Omega} F(u, v) d x \\
& <\lambda_{*} \alpha M C_{1}\|(u, v)\|^{\alpha} \\
& \leq\|(u, v)\|^{\alpha}\left(\frac{p-\beta}{\alpha-\beta}\right)\left[\frac{\alpha-p}{\beta M C_{2}(\alpha-\beta)}\right]^{\frac{\alpha-p}{p-\beta}},
\end{aligned}
$$

and, by (2.11),

$$
0 \leq \lambda \alpha \int_{\Omega} F(u, v) d x<b_{(u, v)}\left(t_{2}^{*}\right) \quad \text { for } 0<\lambda<\lambda_{*} .
$$

Hence, there is a unique $t_{3}=t_{3}(u, v) \in\left(0, t_{2}^{*}\right)$ such that

$$
b_{(u, v)}\left(t_{3}\right)=\lambda \alpha \int_{\Omega} F(u, v) d x \quad \text { and } \quad b_{(u, v)}^{\prime}\left(t_{3}\right)>0 .
$$


Clearly $\left(t_{3} u, t_{3} v\right) \neq(0,0)$, and $\left(t_{3} u, t_{3} v\right) \in \mathcal{N}_{\lambda}$ since

$$
\begin{aligned}
I_{\lambda}\left(t_{3} u, t_{3} v\right) & =t_{3}^{p}\|(u, v)\|^{p}-\lambda \alpha t_{3}^{\alpha} \int_{\Omega} F(u, v) d x-\beta t_{3}^{\beta} \int_{\partial \Omega} G(u, v) d s \\
& =t_{3}^{\alpha}\left(b_{(u, v)}(t)-\lambda \alpha \int_{\Omega} F(u, v) d x\right)=0 .
\end{aligned}
$$

It follows from (2.2) that

$$
\begin{aligned}
\left\langle I_{\lambda}^{\prime}\left(t_{3} u, t_{3} v\right),\left(t_{3} u, t_{3} v\right)\right\rangle & =(p-\alpha) t_{3}^{p}\|(u, v)\|^{p}-\beta(\beta-\alpha) t_{3}^{\beta} \int_{\partial \Omega} G(u, v) d s \\
& =t_{3}^{\alpha+1} b_{(u, v)}^{\prime}\left(t_{3}\right)>0 .
\end{aligned}
$$

Thus $\left(t_{3} u, t_{3} v\right) \in \mathcal{N}_{\lambda}^{+}$. Moreover,

$$
\begin{aligned}
\frac{d}{d t} \mathcal{J}_{\lambda}(t u, t v) & =t^{p-1}\|(u, v)\|^{p}-\lambda \alpha t^{\alpha-1} \int_{\Omega} F(u, v) d x-\beta t^{\beta-1} \int_{\partial \Omega} G(u, v) d s \\
& =t^{\alpha-1}\left(b_{(u, v)}(t)-\lambda \alpha \int_{\Omega} F(u, v) d x\right) .
\end{aligned}
$$

So, $\frac{d}{d t} \mathcal{J}_{\lambda}(t u, t v)=0$ for $t=t_{3} ; \frac{d}{d t} \mathcal{J}_{\lambda}(t u, t v)<0$ for $t \in\left(0, t_{3}\right) ; \frac{d}{d t} \mathcal{J}_{\lambda}(t u, t v)>0$ for $t \in\left(t_{3}, t_{2}^{*}\right)$. Hence, $\mathcal{J}_{\lambda}\left(t_{3} u, t_{3} v\right)=\inf _{0 \leq t \leq t_{2}^{*}} \mathcal{J}_{\lambda}(t u, t v)$.

\section{Main result}

Now we state our main result.

Theorem 3.1. Suppose (H1) and (H2) hold. Then problem (1.1) has at least two nontrivial nonnegative solutions for $\lambda \in\left(0, \lambda_{*}\right)$.

The proof of this theorem will be a consequence of the next two propositions.

Proposition 3.2. Suppose (H1) and (H2) hold and $\lambda \in\left(0, \lambda_{*}\right)$. Then the functional $\mathcal{J}_{\lambda}$ has a minimizer $\left(u_{0}^{+}, v_{0}^{+}\right)$in $\mathcal{N}_{\lambda}^{+}$, and it satisfies

(i) $\mathcal{J}_{\lambda}\left(u_{0}^{+}, v_{0}^{+}\right)=\gamma_{\lambda}^{+}$;

(ii) $\left(u_{0}^{+}, v_{0}^{+}\right)$is a nontrivial nonnegative solution of problem (1.1).

Proof. By Lemma $2.4, \mathcal{J}_{\lambda}$ is coercive and bounded below on $\mathcal{N}_{\lambda}$. By assumption (H1) and Lemma 2.6(ii), $\mathcal{N}_{\lambda}^{+} \neq \emptyset$. Let $\left\{\left(u_{n}, v_{n}\right)\right\}$ be a minimizing sequence for $\mathcal{J}_{\lambda}$ on $\mathcal{N}_{\lambda}^{+}$, i.e., $\lim _{n \rightarrow+\infty} \mathcal{J}_{\lambda}\left(u_{n}, v_{n}\right)=\inf _{(u, v) \in \mathcal{N}_{\lambda}^{+}} \mathcal{J}_{\lambda}(u, v)=\gamma_{\lambda}^{+}<0$. Then, by Lemma 2.4 and the Rellich-Kondrachov theorem, there exist a subsequence of $\left\{\left(u_{n}, v_{n}\right)\right\}$, denoted by itself, and $\left(u_{0}^{+}, v_{0}^{+}\right) \in W$ such that

$\left(u_{n}, v_{n}\right) \rightarrow\left(u_{0}^{+}, v_{0}^{+}\right)$weakly in $W$

$u_{n} \rightarrow u_{0}^{+}, v_{n} \rightarrow v_{0}^{+}$strongly in $L^{\alpha}(\Omega)$ and $L^{\beta}(\partial \Omega)$, respectively. 
Thus, by (2.3),

$$
\begin{aligned}
\int_{\Omega} F\left(u_{n}, v_{n}\right) d x & \rightarrow \int_{\Omega} F\left(u_{0}^{+}, v_{0}^{+}\right) d x \text { as } n \rightarrow+\infty, \\
\int_{\partial \Omega} G\left(u_{n}, v_{n}\right) d s & \rightarrow \int_{\partial \Omega} G\left(u_{0}^{+}, v_{0}^{+}\right) d s \text { as } n \rightarrow+\infty .
\end{aligned}
$$

From the facts that

$$
\mathcal{J}_{\lambda}\left(u_{n}, v_{n}\right)=\frac{\alpha-p}{\alpha p}\left\|\left(u_{n}, v_{n}\right)\right\|^{p}-\frac{\alpha-\beta}{\alpha} \int_{\partial \Omega} G\left(u_{n}, v_{n}\right) d s,
$$

and

it follows that

$$
\mathcal{J}_{\lambda}\left(u_{n}, v_{n}\right) \rightarrow \gamma_{\lambda}^{+}<0 \text { as } n \rightarrow+\infty
$$

$$
\int_{\partial \Omega} G\left(u_{0}^{+}, v_{0}^{+}\right) d s>0 .
$$

In particular, $\left(u_{0}^{+}, v_{0}^{+}\right) \neq(0,0)$. Now, we prove that $\left(u_{n}, v_{n}\right) \rightarrow\left(u_{0}^{+}, v_{0}^{+}\right)$ strongly in $W$. Suppose otherwise, then

$$
\left\|\left(u_{0}^{+}, v_{0}^{+}\right)\right\|<\lim _{n \rightarrow+\infty}\left\|\left(u_{n}, v_{n}\right)\right\|,
$$

and

$$
\mathcal{J}_{\lambda}\left(u_{0}, v_{0}\right)<\lim _{n \rightarrow \infty} \mathcal{J}_{\lambda}\left(u_{n}, v_{n}\right)=\gamma_{\lambda}^{+} .
$$

Since $\int_{\partial \Omega} G\left(u_{0}^{+}, v_{0}^{+}\right) d s>0$, by Lemma 2.6(ii), there exists a unique $t_{3}=$ $t_{3}\left(u_{0}^{+}, v_{0}^{+}\right) \in\left(0, t_{2}^{*}\left(u_{0}^{+}, v_{0}^{+}\right)\right)$such that $\left(t_{3} u_{0}^{+}, t_{3} v_{0}^{+}\right) \in \mathcal{N}_{\lambda}^{+}$and $\mathcal{J}_{\lambda}\left(t_{3} u_{0}^{+}, t_{3} v_{0}^{+}\right)=$ $\inf _{0 \leq t \leq t_{2}^{*}\left(u_{0}^{+}, v_{0}^{+}\right)} \mathcal{J}_{\lambda}\left(t u_{0}^{+}, t v_{0}^{+}\right)$. Furthermore,

$$
\frac{d}{d t} \mathcal{J}_{\lambda}\left(t u_{0}^{+}, t v_{0}^{+}\right)<0 \quad \text { for } t \in\left(0, t_{3}\right)
$$

Recall that $b_{(u, v)}(t)=t^{p-\alpha}\|(u, v)\|^{p}-\beta t^{\beta-\alpha} \int_{\partial \Omega} G(u, v) d s$ for $t>0$. Then

$$
b_{\left(u_{0}^{+}, v_{0}^{+}\right)}\left(t_{3}\right)=\lambda \alpha \int_{\Omega} F\left(u_{0}^{+}, v_{0}^{+}\right) d x .
$$

By (3.1), (3.2) and (3.5),

$$
\begin{aligned}
& \lim _{n \rightarrow+\infty}\left(b_{\left(u_{n}, v_{n}\right)}\left(t_{3}\right)-\lambda \alpha \int_{\Omega} F\left(u_{n}, v_{n}\right) d x\right) \\
= & \lim _{n \rightarrow+\infty}\left(t_{3}^{p-\alpha}\left\|\left(u_{n}, v_{n}\right)\right\|^{p}-\beta t_{3}^{\beta-\alpha} \int_{\partial \Omega} G\left(u_{n}, v_{n}\right) d s-\lambda \alpha \int_{\Omega} F\left(u_{n}, v_{n}\right) d x\right) \\
= & t_{3}^{p-\alpha} \lim _{n \rightarrow+\infty}\left\|\left(u_{n}, v_{n}\right)\right\|^{p}-\beta t_{3}^{\beta-\alpha} \int_{\partial \Omega} G\left(u_{0}^{+}, v_{0}^{+}\right) d s-\lambda \alpha \int_{\Omega} F\left(u_{0}^{+}, v_{0}^{+}\right) d x \\
> & t_{3}^{p-\alpha}\left\|\left(u_{0}^{+}, v_{0}^{+}\right)\right\|^{p}-\beta t_{3}^{\beta-\alpha} \int_{\partial \Omega} G\left(u_{0}^{+}, v_{0}^{+}\right) d s-\lambda \alpha \int_{\Omega} F\left(u_{0}^{+}, v_{0}^{+}\right) d x \\
= & b_{\left(u_{0}^{+}, v_{0}^{+}\right)}\left(t_{3}\right)-\lambda \alpha \int_{\Omega} F\left(u_{0}^{+}, v_{0}^{+}\right) d x=0,
\end{aligned}
$$


which implies that, for $n$ large enough,

$$
b_{\left(u_{n}, v_{n}\right)}\left(t_{3}\right)>\lambda \alpha \int_{\Omega} F\left(u_{n}, v_{n}\right) d x .
$$

On the other hand, since $\left(u_{n}, v_{n}\right) \in \mathcal{N}_{\lambda}^{+}$, by $(2.2)$,

$$
\int_{\partial \Omega} G\left(u_{n}, v_{n}\right) d s>\frac{(\alpha-p)\left\|\left(u_{n}, v_{n}\right)\right\|^{p}}{\beta(\alpha-\beta)},
$$

which implies that $t_{2}^{*}\left(u_{n}, v_{n}\right)>1$ by Lemma 2.6(ii). Moreover, we obtain

$$
b_{\left(u_{n}, v_{n}\right)}(1)=\left\|\left(u_{n}, v_{n}\right)\right\|^{p}-\beta \int_{\partial \Omega} G\left(u_{n}, v_{n}\right) d s=\lambda \alpha \int_{\Omega} F\left(u_{n}, v_{n}\right) d x,
$$

and $b_{\left(u_{n}, v_{n}\right)}(t)$ is increasing for $t \in\left(0, t_{2}^{*}\left(u_{n}, v_{n}\right)\right)$. Thus

$$
b_{\left(u_{n}, v_{n}\right)}(t) \leq b_{\left(u_{n}, v_{n}\right)}(1)=\lambda \alpha \int_{\Omega} F\left(u_{n}, v_{n}\right) d x \text { for all } t \in(0,1] .
$$

For $n$ sufficiently large, by (3.6) and (3.7),

$$
1<t_{3}<t_{2}^{*}\left(u_{0}^{+}, v_{0}^{+}\right)
$$

By (3.4) and (3.8),

$$
\mathcal{J}_{\lambda}\left(t_{3} u_{0}^{+}, t_{3} v_{0}^{+}\right)<\mathcal{J}_{\lambda}\left(u_{0}^{+}, v_{0}^{+}\right),
$$

which contradicts $\left(t_{3} u_{0}^{+}, t_{3} v_{0}^{+}\right) \in \mathcal{N}_{\lambda}^{+}$by (3.3). Hence

$$
\left(u_{n}, v_{n}\right) \rightarrow\left(u_{0}^{+}, v_{0}^{+}\right) \text {strongly in } W
$$

and

$$
\mathcal{J}_{\lambda}\left(u_{n}, v_{n}\right) \rightarrow \mathcal{J}_{\lambda}\left(u_{0}^{+}, v_{0}^{+}\right)=\gamma_{\lambda}^{+} \text {as } n \rightarrow+\infty \text {. }
$$

By Lemma 2.2, $\left(u_{0}^{+}, v_{0}^{+}\right) \in \mathcal{N}_{\lambda}^{+}$and $\left(u_{0}^{+}, v_{0}^{+}\right)$is a local minimizer for $\mathcal{J}_{\lambda}$ on $\mathcal{N}_{\lambda}$. Since $\mathcal{J}_{\lambda}\left(u_{0}^{+}, v_{0}^{+}\right)=\mathcal{J}_{\lambda}\left(\left|u_{0}^{+}\right|,\left|v_{0}^{+}\right|\right)$and $\left(\left|u_{0}^{+}\right|,\left|v_{0}^{+}\right|\right) \in \mathcal{N}_{\lambda}^{+}$, by Lemma 2.3, we may assume $\left(u_{0}^{+}, v_{0}^{+}\right)$is a nontrivial nonnegative solution of (1.1), and thus the proof is complete.

Proposition 3.3. Suppose (H1) and (H2) hold and $\lambda \in\left(0, \lambda_{*}\right)$. Then the functional $\mathcal{J}_{\lambda}$ has a minimizer $\left(u_{0}^{-}, v_{0}^{-}\right)$in $\mathcal{N}_{\lambda}^{-}$and it satisfies

(i) $\mathcal{J}_{\lambda}\left(u_{0}^{-}, v_{0}^{-}\right)=\gamma_{\lambda}^{-}$;

(ii) $\left(u_{0}^{-}, v_{0}^{-}\right)$is a nontrivial nonnegative solution of problem (1.1).

Proof. By assumption (H1) and Lemma 2.6(i), $\mathcal{N}_{\lambda}^{-} \neq \emptyset$. Let $\left\{\left(u_{n}, v_{n}\right)\right\}$ be a minimizing sequence for $\mathcal{J}_{\lambda}$ on $\mathcal{N}_{\lambda}^{-}$, i.e.,

$$
\lim _{n \rightarrow+\infty} \mathcal{J}_{\lambda}\left(u_{n}, v_{n}\right)=\inf _{(u, v) \in \mathcal{N}_{\lambda}^{-}} \mathcal{J}_{\lambda}(u, v) .
$$

Then by Lemma 2.4 and the Rellich-Kondrachov theorem, there exists a subsequence of $\left\{\left(u_{n}, v_{n}\right)\right\}$, denoted by itself, and $\left(u_{0}^{-}, v_{0}^{-}\right) \in W$ such that

$$
\begin{aligned}
& \left(u_{n}, v_{n}\right) \rightarrow\left(u_{0}^{-}, v_{0}^{-}\right) \text {weakly in } W \\
& u_{n} \rightarrow u_{0}^{-}, v_{n} \rightarrow v_{0}^{-} \text {strongly in } L^{\alpha}(\Omega) \text { and } L^{\beta}(\partial \Omega) \text {, respectively. }
\end{aligned}
$$


Thus, by (2.3),

$$
\begin{aligned}
\int_{\Omega} F\left(u_{n}, v_{n}\right) d x & \rightarrow \int_{\Omega} F\left(u_{0}^{-}, v_{0}^{-}\right) d x \text { as } n \rightarrow+\infty, \\
\int_{\partial \Omega} G\left(u_{n}, v_{n}\right) d s & \rightarrow \int_{\partial \Omega} G\left(u_{0}^{-}, v_{0}^{-}\right) d s \text { as } n \rightarrow+\infty .
\end{aligned}
$$

Moreover, by (2.2),

$$
\int_{\Omega} F\left(u_{n}, v_{n}\right) d x>\frac{p-\beta}{\lambda_{*} \alpha(\alpha-\beta)}\left\|\left(u_{n}, v_{n}\right)\right\|^{p} .
$$

By (2.9) and (3.9), there exists a positive number $D$ such that

$$
\int_{\Omega} F\left(u_{n}, v_{n}\right) d x>D
$$

which implies

$$
\int_{\Omega} F\left(u_{0}^{-}, v_{0}^{-}\right) d x \geq D
$$

Now we prove that $\left(u_{n}, v_{n}\right) \rightarrow\left(u_{0}^{-}, v_{0}^{-}\right)$strongly in $W$. Suppose otherwise, then

$$
\left\|\left(u_{0}^{-}, v_{0}^{-}\right)\right\|<\lim _{n \rightarrow+\infty}\left\|\left(u_{n}, v_{n}\right)\right\| .
$$

By Lemma 2.6(i) and (3.10), there exists a unique $t_{2}=t_{2}\left(u_{0}, v_{0}\right)$ such that $t_{2}>t_{1}^{*}\left(u_{0}, v_{0}\right),\left(t_{2} u_{0}^{-}, t_{2} v_{0}^{-}\right) \in \mathcal{N}_{\lambda}^{-}$and

$$
\mathcal{J}_{\lambda}\left(t_{2} u_{0}^{-}, t_{2} v_{0}^{-}\right)=\sup _{t \geq 0} \mathcal{J}_{\lambda}\left(t u_{0}^{-}, t v_{0}^{-}\right)
$$

Since $\left(u_{n}, v_{n}\right) \in \mathcal{N}_{\lambda}^{-}, t_{1}^{*}\left(u_{n}, v_{n}\right)<1$ and $a_{\left(u_{n}, v_{n}\right)}(1)=\beta \int_{\partial \Omega} G\left(u_{n}, v_{n}\right) d s$ for all $n \in \mathbb{N}$. Thus $t_{2}\left(u_{n}, v_{n}\right)=1$ and $\mathcal{J}_{\lambda}\left(u_{n}, v_{n}\right) \geq \mathcal{J}_{\lambda}\left(t_{2} u_{n}, t_{2} v_{n}\right)$ by Lemma 2.6(i). On the other hand, by (3.11),

$$
\mathcal{J}_{\lambda}\left(t_{2} u_{0}^{-}, t_{2} v_{0}^{-}\right)<\lim _{n \rightarrow+\infty} \mathcal{J}_{\lambda}\left(t_{2} u_{n}, t_{2} v_{n}\right)
$$

and thus

$$
\mathcal{J}_{\lambda}\left(t_{2} u_{0}^{-}, t_{2} v_{0}^{-}\right)<\lim _{n \rightarrow+\infty} \mathcal{J}_{\lambda}\left(u_{n}, v_{n}\right)=\gamma_{\lambda}^{-} .
$$

This is a contradiction to the fact that $\left(t_{2} u_{0}^{-}, t_{2} v_{0}^{-}\right) \in \mathcal{N}_{\lambda}^{-}$. Hence

$$
\left(u_{n}, v_{n}\right) \rightarrow\left(u_{0}^{-}, v_{0}^{-}\right) \text {strongly in } W \text { as } n \rightarrow+\infty \text {. }
$$

This implies

$$
\mathcal{J}_{\lambda}\left(u_{n}, v_{n}\right) \rightarrow \mathcal{J}_{\lambda}\left(u_{0}^{-}, v_{0}^{-}\right)=\gamma_{\lambda}^{-} \text {as } n \rightarrow+\infty \text {. }
$$

By Lemma 2.2, $\left(u_{0}^{-}, v_{0}^{-}\right) \in \mathcal{N}_{\lambda}^{-}$and $\left(u_{0}^{-}, v_{0}^{-}\right)$is a local minimizer for $\mathcal{J}_{\lambda}$ on $\mathcal{N}_{\lambda}$. Since $\mathcal{J}_{\lambda}\left(u_{0}^{-}, v_{0}^{-}\right)=\mathcal{J}_{\lambda}\left(\left|u_{0}^{-}\right|,\left|v_{0}^{-}\right|\right)$and $\left(\left|u_{0}^{-}\right|,\left|v_{0}^{-}\right|\right) \in \mathcal{N}_{\lambda}^{-}$, by Lemma 2.3, we may assume that $\left(u_{0}^{-}, v_{0}^{-}\right)$is a nontrivial nonnegative solution of (1.1), and thus the proof is complete. 
Proof of Theorem 3.1. By Propositions 3.2 and 3.3, we obtain problem (1.1) has two nontrivial nonnegative solutions $\left(u_{0}^{+}, v_{0}^{+}\right)$and $\left(u_{0}^{-}, v_{0}^{-}\right)$such that $\left(u_{0}^{+}\right.$, $\left.v_{0}^{+}\right) \in \mathcal{N}_{\lambda}^{+}$and $\left(u_{0}^{-}, v_{0}^{-}\right) \in \mathcal{N}_{\lambda}^{-}$. Since $\mathcal{N}_{\lambda}^{+} \cap \mathcal{N}_{\lambda}^{-}=\emptyset,\left(u_{0}^{+}, v_{0}^{+}\right)$and $\left(u_{0}^{-}, v_{0}^{-}\right)$ are distinct, and thus the proof is complete.

Acknowledgments. This work was done when the authors visited College of William and Mary in 2012. They would like to thank Department of Mathematics, College of William and Mary for warm hospitality, and thank Professor Junping Shi for constant encouragement and helpful discussions.

\section{References}

[1] R. P. Agarwal, M. B. Ghaemi, and S. Saiedinezhad, The Nehari manifold for the degenerate p-Laplacian quasilinear elliptic equations, Adv. Math. Sci. Appl. 20 (2010), no. 1, $37-50$

[2] C. O. Alves and A. El Hamidi, Nehari manifold and existence of positive solutions to a class of quasilinear problems, Nonlinear Anal. 60 (2005), no. 4, 611-624.

[3] A. Anane, Simplicité et isolation de la première valeur propre du p-Laplacien avec poids, C. R. Acad. Sci. Paris Sér. I Math. 305 (1987), no. 16, 725-728.

[4] A. Anane and J.-P. Gossez, Strongly nonlinear elliptic problems near resonance: a variational approach, Comm. Partial Differential Equations 15 (1990), no. 8, 1141-1159.

[5] G. Barles, Remarks on uniqueness results of the first eigenvalue of the p-Laplacian, Ann. Fac. Sci. Toulouse Math. (5) 9 (1988), no. 1, 65-75.

[6] A. Bechah, K. Chaïb, and F. de Thélin, Existence and uniqueness of positive solution for subhomogeneous elliptic problems in $\mathbf{R}^{N}$, Rev. Mat. Apl. 21 (2000), no. 1-2, 1-17.

[7] M.-F. Bidaut-Véron and T. Raoux, Propriétés locales des solutions d'un système elliptique non linéaire, C. R. Acad. Sci. Paris Sér. I Math. 320 (1995), no. 1, 35-40.

[8] L. Boccardo and D. Guedes de Figueiredo, Some remarks on a system of quasilinear elliptic equations, Nonlinear Differential Equations Appl. 9 (2002), no. 3, 309-323.

[9] J. F. Bonder and J. D. Rossi, Existence results for the p-Laplacian with nonlinear boundary conditions, J. Math. Anal. Appl. 263 (2001), no. 1, 195-223.

[10] K. J. Brown, The Nehari manifold for a semilinear elliptic equation involving a sublinear term, Calc. Var. Partial Differential Equations 22 (2005), no. 4, 483-494.

[11] K. J. Brown and T.-F. Wu, A fibering map approach to a semilinear elliptic boundary value problem, Electron. J. Differential Equations 2007 (2007), no. 69, 1-9.

$[12] ـ$, A semilinear elliptic system involving nonlinear boundary condition and signchanging weight function, J. Math. Anal. Appl. 337 (2008), no. 2, 1326-1336.

[13] J. Chabrowski, On multiple solutions for nonhomogeneous system of elliptic equations, Rev. Mat. Univ. Complut. Madrid 9 (1996), no. 1, 207-234.

[14] C.-Y. Chen, Y.-C. Kuo, and T.-F. Wu, The Nehari manifold for a Kirchhoff type problem involving sign-changing weight functions, J. Differential Equations 250 (2011), no. 4, 1876-1908.

[15] C.-Y. Chen and T.-F. Wu, The Nehari manifold for indefinite semilinear elliptic systems involving critical exponent, Appl. Math. Comput. 218 (2012), no. 22, 10817-10828.

[16] M. Chipot, M. Chlebík, M. Fila, and I. Shafrir, Existence of positive solutions of a semilinear elliptic equation in $\mathbb{R}_{+}^{n}$ with a nonlinear boundary condition, J. Math. Anal. Appl. 223 (1998), no. 2, 429-471.

[17] M. Chipot, I. Shafrir, and M. Fila, On the solutions to some elliptic equations with nonlinear Neumann boundary conditions, Adv. Differential Equations 1 (1996), no. 1, 91-110. 
[18] P. Clément, J. Fleckinger, E. Mitidieri, and F. de Thélin, Existence of positive solutions for a nonvariational quasilinear elliptic system, J. Differential Equations 166 (2000), no. 2, 455-477.

[19] F. de Thélin, Quelques résultats d'existence et de non-existence pour une EDP elliptique non linéaire, C. R. Acad. Sci. Paris Sér. I Math. 299 (1984), no. 18, 911-914.

[20] M. A. del Pino and R. F. Manásevich, Global bifurcation from the eigenvalues of the p-Laplacian, J. Differential Equations 92 (1991), no. 2, 226-251.

[21] J. I. Díaz, Nonlinear Partial Differential Equations and Free Boundaries. Vol. I, Volume 106 of Research Notes in Mathematics, Pitman (Advanced Publishing Program), Boston, MA, 1985.

[22] E. DiBenedetto, Degenerate Parabolic Equations, Springer-Verlag, New York, 1993.

[23] P. Drábek, Nonlinear eigenvalue problem for $p$-Laplacian in $\mathbf{R}^{N}$, Math. Nachr. 173 (1995), 131-139.

[24] P. Drábek and Y. X. Huang, Bifurcation problems for the p-Laplacian in $\mathbf{R}^{N}$, Trans. Amer. Math. Soc. 349 (1997), no. 1, 171-188.

[25] P. Drábek and S. I. Pohozaev, Positive solutions for the p-Laplacian: application of the fibering method, Proc. Roy. Soc. Edinburgh Sect. A 127 (1997), no. 4, 703-726.

[26] J. F. Escobar, Uniqueness theorems on conformal deformation of metrics, Sobolev inequalities, and an eigenvalue estimate, Comm. Pure Appl. Math. 43 (1990), no. 7, 857-883.

[27] P. Felmer, R. F. Manásevich, and F. de Thélin, Existence and uniqueness of positive solutions for certain quasilinear elliptic systems, Comm. Partial Differential Equations 17 (1992), no. 11-12, 2013-2029.

[28] J. Garcia-Azorero, I. Peral, and J. D. Rossi, A convex-concave problem with a nonlinear boundary condition, J. Differential Equations 198 (2004), no. 1, 91-128.

[29] Y. Li, Asymptotic behavior of positive solutions of equation $\Delta u+K(x) u^{p}=0$ in $\mathbf{R}^{n}$, J. Differential Equations 95 (1992), no. 2, 304-330.

[30] P. Lindqvist, On the equation $\operatorname{div}\left(|\nabla u|^{p-2} \nabla u\right)+\lambda|u|^{p-2} u=0$, Proc. Amer. Math. Soc. 109 (1990), no. 1, 157-164.

[31] Z. Nehari, On a class of nonlinear second-order differential equations, Trans. Amer. Math. Soc. 95 (1960), 101-123.

[32] _ Characteristic values associated with a class of non-linear second-order differential equations, Acta Math. 105 (1961), 141-175.

[33] W.-M. Ni and J. Serrin, Existence and non-existence theorems for ground states of quasilinear partial differential equations. The anomalous case, Rome, Acc. Naz. dei Lincei, Atti dei Convegni 77 (1986), 231-257.

[34] M. Ôtani, On certain second order ordinary differential equations associated with Sobolev-Poincaré-type inequalities, Nonlinear Anal. 8 (1984), no. 11, 1255-1270.

[35] _ Existence and nonexistence of nontrivial solutions of some nonlinear degenerate elliptic equations, J. Funct. Anal. 76 (1988), no. 1 140-159.

[36] D. Pierotti and S. Terracini, On a Neumann problem with critical exponent and critical nonlinearity on the boundary, Comm. Partial Differential Equations 20 (1995), no. 7-8, $1155-11875$.

[37] S. H. Rasouli and G. A. Afrouzi, The Nehari manifold for a class of concave-convex elliptic systems involving the p-Laplacian and nonlinear boundary condition, Nonlinear Anal. 73 (2010), no. 10, 3390-3401.

[38] G. Tarantello, On nonhomogeneous elliptic equations involving critical Sobolev exponent, Ann. Inst. H. Poincaré Anal. Non Linéaire 9 (1992), no. 3, 281-304.

[39] P. Tolksdorf, Regularity for a more general class of quasilinear elliptic equations, J. Differential Equations 51 (1984), no. 1, 126-150.

[40] T.-F. Wu, On semilinear elliptic equations involving concave-convex nonlinearities and sign-changing weight function, J. Math. Anal. Appl. 318 (2006), no. 1, 253-270. 
[41] _ Multiple positive solutions for semilinear elliptic systems with nonlinear boundary condition, Appl. Math. Comput. 189 (2007), no. 2, 1712-1722.

[42] Z. Wu, J. Zhao, J. Yin, and H. Li, Nonlinear Diffusion Equations, World Scientific Publishing Co. Inc., River Edge, NJ, 2001.

JuN ZHOU

School of Mathematics and Statistics

SOUTHWEST UNIVERSITY

Chongqing, 400715, P. R. China

E-mail address: jzhouwm@gmail.com

Chan-Gyun Kim

Department of Mathematics

College of William and Mary

Williamsburg, Virginia, 23187-8795, USA

E-mail address: cgkim75@gmail.com 